\title{
CÁC YẾU TỐ TÁC ĐộNG ĐẾN HIỆ QUẢ CỦA CÁC DOANH NGHIỆP BẤT ĐộNG SẢN NIỂM YẾT TẠI VIỆT NAM
}

\author{
NGUYỄN THỊ MỸ PHƯợNG \\ Khoa Tài chính - Ngân hàng, Truờng Đại học Công nghiệp thành phố Hồ Chí Minh \\ nguyenthimyphuong@iuh.edu.vn
}

Tóm tắt. Bài viết này ước tính hiệu quả và nghiên cứu các yếu tố tác động đến hiệu quả của các doanh nghiệp bất động sản niêm yết tại Việt Nam (DNBĐSVN) trong thời kỳ 2010-2019. Đầu tiên, nghiên cứu đo lường mức độ hiệu quả của các DNBĐSVN bằng cách sử dụng phương pháp phân tích bao dữ liệu (Data Envelopment Analysis - DEA). Sau đó, nghiên cứu sử dụng kết hợp hai mô hình Tobit và Bayesian Model Averaging (BMA) để xác định các yếu tố tác động đến hiệu quả của các DNBĐSVN. Kết quả nghiên cứu cho thấy hiệu quả theo quy mô trung bình của các DNBĐSVN ở mức $93,01 \%$, với CRSTE là $80,50 \%$ và VRSTE là $86,61 \%$, phản ánh rằng các DNBĐSVN chưa sử dụng tối đa các nguồn lực đầu vào. Kết quả mô hình Tobit và $\mathrm{BMA}$ chỉ ra rằng qui mô doanh nghiệp, vòng quay tài sản, khả năng sinh lời, sở hữu nước ngoài, giá bất động sản, chỉ số tiếp cận đất đai, tăng trưởng kinh tế, tính dễ tổn thương của khu vực ngân hàng và hiệu quả Chính phủ có tác động cùng chiều, trong khi đó rủi ro hệ thống, cấu trúc vốn, lãi suất và lạm phát có tác động ngược chiều đến hiệu quả của các DNBĐSVN.

Từ khóa: Hiệu quả, doanh nghiệp bất động sản, DEA, TOBIT

\section{DETERMINANTS OF EFFICIENCY OF LISTED REAL ESTATE FIRMS IN VIETNAM}

Abstract. This paper estimates efficiency and examine factors that influence the efficiency of listed Vietnamese real estate firms for the period of 2010-2019. In the first stage, we estimate the efficiency level of listed real estate firms by employing the data envelopment analysis (DEA) approach. In the second stage, the Tobit Model and Bayesian Model Averaging (BMA) were estimated to determine factors that influence efficiency of obtained in the first stage. The results indicate that the average scale efficiency is $93,01 \%$, with CRSTE at 80,50\% and VRSTE at 86,61\%, showing that the Vietnamese real estate firms have not used the maximum input resources. The Tobit and BMA regression result show that size, total assets turnover, profitability, foreign ownership, real estate price, land access, economic growth, banking sector fragility and Government efficiency share positively affects the efficiency of Vietnamese real estate firms while systematic risk, capital structure, interest rates and inflation negatively affect the efficiency as predicted.

Keywords: Efficiency, real estate firm, DEA, TOBIT

\section{GIỚI THIỆU}

Thị trường bất động sản (BĐS) Việt Nam hình thành từ năm 1993 sau khi Quốc hội thông qua Luật Đất đai đầu tiên và Pháp lệnh Nhà ở. Trong gần 30 năm hình thành và phát triển, thị trường $B Đ S$ Việt Nam đã tăng trưởng mạnh về qui mô, phạm vi, số lượng và chất lượng. Trong bối cảnh tăng trưởng của thị trường $\mathrm{B} Đ S$ thì hoạt động kinh doanh của các doanh nghiệp BĐS Việt Nam cũng ngày càng phát triển. Tính đến năm 2019 , có 15.000 doanh nghiệp BĐS đang hoạt động, đã đóng góp 4,51\% GDP và $0,6 \%$ tổng số việc làm ở Việt Nam (GSO, 2019). Kết quả này cho thây, hiệu quả của các doanh nghiệp BĐS Việt Nam có ảnh hưởng trực tiếp đến sự phát triển của nền kinh tế quốc gia và sự ổn định xã hội. Tuy nhiên, trong những năm gần đây, sự tăng giá BĐS mạnh mẽ tại các khu vực như Thành phố Hồ Chí Minh, Hải Phòng, Đà Nẵng, Khánh Hòa đã gây ra mối lo ngại về tình trạng "bong bóng BĐS" sẽ tiếp tục tái diê̂n theo chu kỳ 10 năm và điều này sẽ có tác động đến hiệu quả của khu vực doanh nghiệp BĐS Việt Nam. Kết thúc năm 2019, thị trường BĐS Việt Nam có sự sụt giảm mạnh mẽ cả về nguồn cung và lượng giao dịch với tổng lượng cung căn hộ đạt 107.284 sản phẩm, giảm chỉ bằng $62 \%$ so với năm 2018 ; lượng giao dịch đạt 72.828 sản phẩm, chỉ bằng 65\% so với năm 2018 (VARS, 2020). Cũng trong năm 2019, có đến 598 doanh nghiệp BĐS đăng ký tạm dừng hoạt động, tăng 36,8\%; và có đến 686 doanh nghiệp $\mathrm{B} Đ S$ giải thể, tăng 39,4\% so với năm 2018, đứng đầu so với các ngành sản xuất kinh doanh khác (MPI, 2019). Thực trạng này phản ánh rằng hiệu quả của 
các doanh nghiệp BĐS Việt Nam nói chung và các doanh nghiệp bất động sản niêm yết tại Việt Nam (DNBĐSVN) nói riêng đang sụt giảm và một câu hỏi được đặt ra là làm thế nào để nâng cao hiệu quả tại doanh nghiệp này trong bối cảnh hiện nay? Theo đó, để nâng cao hiệu quả của các DNBĐSVN, đòi hỏi phải xác định được các yếu tố tác động đến hiệu quả của các doanh nghiệp này. Chính vì vậy, chủ đề nghiên cứu về các yếu tố tác động đến hiệu quả các DNBĐSVN nhằm đưa ra những hàm ý góp phần nâng cao hiệu quả cho các DNBĐSVN hiện nay là vô cùng cấp thiết.

\section{CƠ SỞ LÝ THUYẾT VÀ TỔNG QUAN CÁC NGHIÊN CÚUU TRƯớC}

2.1 Cơ sở lý thuyết

\section{Hiệu quả của doanh nghiệp bất động sản}

Hiệu quả là một chỉ số hiệu suất chung cho tất cả các loại hình doanh nghiệp, trong đó có doanh nghiệp BĐS được hình thành lần đầu tiên trong nghiên cứu của Edgeworth (1881), sau đó là Pareto (1927) và Shephard (1953). Hiệu quả trong kinh tế được hiểu là tỷ lệ tiềm năng tối đa giữa đầu ra và đầu vào của quá trình sản xuất sản phẩm, phản ánh sự phân phối tối ưu các nguồn lực sẵn có để đạt được tiềm năng tối đa (Cvilikas \& Jurkonyte-Dumbliauskiene, 2016). Theo Drucker (1963), hiệu quả có thể được định nghĩa là khả năng của một doanh nghiệp đạt được đầu ra từ mức đầu vào tối thiểu.

Theo Coelli (2005), một doanh nghiệp có hiệu quả hơn so với một doanh nghiệp khác nếu nó có thể cung cấp hàng hóa và dịch vụ nhiều hơn cho xã hội mà không cần sử dụng nhiều nguồn lực hơn đơn vị khác. Theo đó, hiệu quả kỹ thuật là khả năng sử dụng đầu vào tối thiểu để sản xuất một đầu ra cho trước hay khả năng tạo ra đầu ra tối đa từ một đầu vào cho trước. Nếu một doanh nghiệp đang hoạt động ở quy mô không phù hợp (quá nhỏ hoặc quá lớn) thì được gọi là quy mô không hiệu quả. Trong khi đó, một doanh nghiệp sử dụng các yếu tố đầu vào và đầu ra không đúng tỷ lệ được gọi là sự kết hợp kém hiệu quả (Cummins \& cộng sự,1999). Do đó, có thể kết luận rằng doanh nghiệp đạt hiệu quả kỹ thuật nhưng vẫn có thể cải thiện năng suất bằng cách khai thác quy mô kinh tế.

Để đo lường hiệu quả của các doanh nghiệp BĐS, các nghiên cứu trước của Ran \& Xu (2013), Xiao (2014), Wang \& Jiang (2015) và Abdullah \& cộng sự (2019) đều sử dụng cách tiếp cận DEA do kỹ thuật vượt trội của phương pháp này so với các phương pháp khác trong việc tính toán điểm hiệu quả tổng thể trên các ngành công nghiệp khác nhau (Kumar \& Arora, 2011). Charnes \& cộng sự (1978) đã phát triển DEA như một kỹ thuật toán học dựa trên lập trình tuyến tính để đo lường hiệu quả tương đối của các DMU với biên sản xuất cố định theo quy mô (Constant returns to scale - CRS). Sau đó, Banker \& cộng sự (1984) đã phát triển thành mô hình với biên sản xuất biến đổi theo quy mô (Variable returns to scale - VRS).

\section{Các yếu tố tác động đến hiệu quả của doanh nghiệp bất động sản}

Các nghiên cứu trước của Abdullah \& cộng sự (2019), Wei \& cộng sự (2004), Saul \& cộng sự (2008), You \& Zi, (2007), Ran \& Xu (2013), Xiao (2014), Wang \& Jiang (2015) đã chỉ ra các yếu tố tác động đến hiệu quả doanh nghiệp nói chung và doanh nghiệp BĐS nói riêng bao gồm hai nhóm: (i) Nhóm yếu tố đặc thù doanh nghiệp: Qui mô doanh nghiệp, khả năng thanh toán, vòng quay tài sản, khả năng sinh lời, tốc độ tăng trưởng, cấu trúc vốn, tuổi của doanh nghiệp, sở hữu nhà nước, sở hữu nước ngoài và (ii) Nhóm yếu tố vĩ mô: Tăng trưởng kinh tế, lãi suất, lạm phát, tỷ giá. Tuy nhiên, theo quan điểm của Klimczak (2010), Lieser \& Groh (2014) thì hiệu quả của các doanh nghiệp BĐS còn chịu tác động của nhóm yếu tố đặc thù ngành. Do đó, dựa trên các nghiên cứu trước và lý thuyết về chi phí - lợi ích cho thấy các nhóm yếu tố tác động đến hiệu quả của các doanh nghiệp BĐS, bao gồm nhóm yếu tố đặc thù doanh nghiệp, nhóm yếu tố vĩ mô và nhóm yếu tố đặc thù ngành.

\subsection{Tổng quan các nghiên cứu trước}

Liên quan đến chủ đề nghiên cứu về hiệu quả doanh nghiệp phi tài chính nói chung, tính đến nay đã có nhiều nghiên cứu trong và ngoài nước như các nghiên cứu về ảnh hưởng của cấu trúc vốn đến hiệu quả hoạt động doanh nghiệp như Khải \& Toàn (2015), Trúc \& Thiên (2015), Ngọc \&Trang (2016), Phúc (2018), Hưng \& Mai (2018), Thủy (2020). Các nghiên cứu về ảnh hưởng của cấu trúc sở hữu đến hiệu quả hoạt động doanh nghiệp như Abdullah \& cộng sự (2019), Wei \& cộng sự (2004), Saul \& cộng sự (2008), Việt \& Mẫn (2016), Cường \& Tiến (2017). Các nghiên cứu của You \& Zi, (2007), Thủy \& cộng sự (2017), Hương (2020) nghiên cứu ảnh hưởng của các yếu tố như khủng hoảng kinh tế, đặc điểm tổng giám đốc điều hành, các nhân tố cấu thành kiểm soát nội bộ và các công ty zombie đến hiệu quả hoạt động doanh nghiệp. Tuy nhiên, có rất ít nghiên cứu tập trung về hiệu quả của các doanh nghiệp BĐS như Abdullah \& cộng sự (2019) đo lường mức độ hiệu quả và xác định các yếu tố tác động đến hiệu quả của các doanh nghiệp BĐS niêm 
yết trên Sở giao dịch chứng khoán Kuala Lumpur; Ran \& Xu (2013), Xiao (2014), Wang \& Jiang (2015) đo lường hiệu quả của các doanh nghiệp BĐS Trung Quốc; Trí (2018) nghiên cứu các nhân tố ảnh hưởng đến hiệu quả kinh doanh của doanh nghiệp BĐS Việt Nam niêm yết trên HSX.

Như vậy, qua lược khảo các nghiên cứu trước cho thấy tại Việt Nam chưa có nghiên cứu nào đồng thời đo lường hiệu quả các DNBĐSVN theo phương pháp DEA và sử dụng kết hợp hai mô hình Tobit \& BMA để xác định các yếu tố tác động đến hiệu quả của các DNBĐSVN. Về phương pháp tiếp cận, hầu hết các nghiên cứu tại Việt Nam khi đánh giá hiệu quả của các doanh nghiệp phi tài chính đều sử dụng chỉ tiêu tỷ suất lợi nhuận/tổng tài sản (Return on assets - ROA) hoặc tỷ suất lợi nhuận/vốn chủ sở hữu (Return on Equity - ROE). Tuy nhiên, ROA hoặc ROE chỉ là một trong những chỉ tiêu tài chính phản ánh khả năng sinh lời của doanh nghiệp, chưa thể đo lường toàn diện thực trạng hiệu quả doanh nghiệp và không đánh giá được mức độ phân bổ các nguồn lực đầu vào (Ross \& cộng sự, 2012). Trong khi đó, phương pháp DEA với những ưu điểm vượt trội có thể đo lường và xếp hạng hiệu quả tổng thể của doanh nghiệp một cách toàn diện và chính xác (Kumar \& Arora, 2011). Ngoài ra, tất cả các nghiên cứu trước về các yếu tố tác động đến hiệu quả của các doanh nghiệp BĐS đều: (i) Chưa tính đến một cách đầy đủ tác động đồng thời của các nhóm yếu tố đặc thù doanh nghiệp, nhóm yếu tố đặc thù ngành và nhóm yếu tố vĩ mô; (ii) Chưa tính đến tác động của các yếu tố giá BĐS, chỉ số tiếp cận đất đai, rủi ro hệ thống, tính dễ tổn thương của khu vực ngân hàng và hiệu quả Chính phủ. Do đó, bài viết này kỳ vọng lấp đầy những khoảng trống nghiên cứu đã nêu, mang lại đóng góp mới về phương pháp tiếp cận tại Việt Nam, bổ sung những bằng chứng thực nghiệm mới vào khung lý thuyết các yếu tố tác động đến hiệu quả doanh nghiệp nói chung và doanh nghiệp BĐS nói riêng tại các nền kinh tế mới nổi như Việt Nam.

\section{PHƯƠNG PHÁP NGHIÊN CÚ̉U VÀ DŨ LIỆU}

Bài viết sử dụng phương pháp nghiên cứu định lượng nhằm mục tiêu xác định các yếu tố ảnh hưởng đến hiệu quả kỹ thuật và hiệu quả theo quy mô của 38 DNBĐSVN trong giai đoạn 2010-2019. Nghiên cứu tiến hành theo hai giai đoạn: (i) Đo lường hiệu quả của các DNBĐSVN theo phương pháp DEA; (ii) Sử dụng mô hình Tobit và BMA để xác định các yếu tố ảnh hưởng đến hiệu quả của các DNBĐSVN.

Về kích thước mẫu nghiên cứu, để phân tích hồi quy Tobit và BMA đảm bảo độ tin cậy, theo Tabachnick \& Fidell (2007), kích thước mẫu phải là: $\mathrm{n} \geq 8 \mathrm{~m}+50$; trong khi đó, theo Harris (1985) thì $\mathrm{n} \geq 104+\mathrm{m}$ (với $\mathrm{n}$ là cỡ mẫu, $\mathrm{m}$ là số biến độc lập trong mô hình).Trong bài viết này, mô hình nghiên cứu có 18 biến độc lập nên kích thước mẫu tính theo quan điểm của Tabachnick \& Fidell (2007) là $n \geq 194$ quan sát, theo Harris (1985) là $n \geq 122$ quan sát. Do đó, cỡ mẫu của nghiên cứu này có tổng số 380 quan sát nên thỏa mãn quy định về cỡ mẫu tối thiểu, vì vậy kết quả nghiên cứu đảm bảo được độ tin cậy về mặt thống kê.

\section{1 Đo lường hiệu quả của các DNBĐSVN}

\section{Phương pháp đo lường hiệu quả của các DNBĐSVN}

Để đo lường hiệu quả của 38 DNBĐSVN trong giai đoạn 2010-2019, tác giả sử dụng cách tiếp cận DEA. Theo Charnes \& cộng sự (1978), DEA là mô hình lập trình toán học được áp dụng cho dữ liệu quan sát cung cấp một cách mới để có được ước tính thực nghiệm về các mối quan hệ như các hàm sản xuất hoặc bề mặt khả năng sản xuất hiệu quả. Cách tiếp cận này cung cấp một giá trị hiệu quả số được xác định một cách khách quan bằng cách sử dụng nhiều đầu vào và đầu ra.

Giả sử rằng có $\mathrm{n}$ đơn vị ra quyết định (DMU) hay DNBĐSVN được đánh giá. Mỗi loại tiêu thụ số lượng đầu vào $\mathrm{i}$ khác nhau và tạo ra $\mathrm{r}$ đầu ra khác nhau, tức là $\mathrm{DMU}_{\mathrm{j}}$ tiêu thụ $\mathrm{x}_{\mathrm{ji}}$ lượng đầu vào để tạo ra số lượng

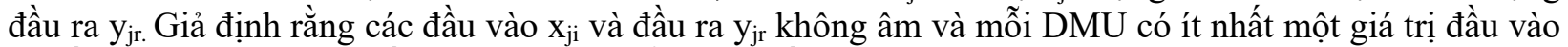
và đầu ra dương. Năng suất của DMU có thể được viết là:

$$
h_{j}=\frac{\sum_{r=1}^{s} u_{r} y_{r j}}{\sum_{i=1}^{m} v_{i} x_{i j}}
$$

Trong công thức (1), $\mathrm{u}$ và $\mathrm{v}$ là các trọng số được gán cho từng đầu vào và đầu ra. Bằng cách sử dụng các kỹ thuật lập trình toán học, DEA gán tối ưu các trọng số theo các ràng buộc sau: Các trọng số cho mỗi $\mathrm{DMU}$ được gán theo ràng buộc rằng không có $\mathrm{DMU}$ nào khác có hiệu suất lớn hơn 1 nếu nó sử dụng cùng các trọng số, ngụ ý rằng DMU hoàn toàn hiệu quả sẽ có giá trị là 1 . 
Trong nghiên cứu này, tác giả tính toán hiệu quả kỹ thuật của cả hai mô hình DEA với biên sản xuất cố định theo quy mô (CRSTE) và biên sản xuất biến đổi theo quy mô (VRSTE) với giả thiết tối thiểu hóa các đầu vào mà không làm giảm sút các đầu ra và tối đa hóa các đầu ra dựa trên các đầu vào có sẵn. Theo đó, hiệu quả theo quy mô (SE) được xác định theo công thức: $\mathrm{SE}=\mathrm{CRSTE} / \mathrm{VRSTE}$.

Các hệ số hiệu quả CRSTE, VRSTE và SE được ước lượng từ mô hình DEA cho giá trị từ 0 đến $1(0 \%$ đến $100 \%$ ) cho thấy mức độ hiệu quả của DNBĐSVN từ hiệu quả thấp nhất đến hiệu quả hoàn toàn.

\section{Dũ̃ liệu các biến trong mồ hình DEA}

Để lựa chọn các biến trong phân tích DEA nhằm đo lường hiệu quả của 38 DNBĐSVN, nghiên cứu dựa trên cách tiếp cận theo quan điểm của Wang \& Jiang (2015). Theo cách tiếp cận này, các biến đầu vào (Input), đầu ra (Output) của các DNBĐSVN được trình bày tại Bảng 1 . Nguồn dữ liệu các biến được thu thập từ báo cáo tài chính (BCTC) của 38 DNBĐSVN trong giai đoạn 2010-2019.

Bảng 1: Các biến đầu vào và đầu ra trong phân tích DEA

\begin{tabular}{|c|c|c|c|}
\hline & Biến & Ký hiệu & Đơn vị tính \\
\hline \multirow{2}{*}{${ }_{0}^{2}$} & $\begin{array}{l}\text { Doanh thu thuần về bán hàng và cung cấp dịch vụ \& } \\
\text { doanh thu hoạt động tài chính }\end{array}$ & Y1 & Triệu đồng \\
\hline & Lợi nhuận sau thuế thu nhập doanh nghiệp & $\mathrm{Y} 2$ & Triệu đồng \\
\hline \multirow{3}{*}{ 富 } & Tổng tài sản & $\mathrm{X} 1$ & Triệu đồng \\
\hline & Giá vốn hàng bán & $\mathrm{X} 2$ & Triệu đồng \\
\hline & $\begin{array}{l}\text { Chi phí tài chính, chi phí bán hàng và chi phí quản lý } \\
\text { doanh nghiệp }\end{array}$ & $\mathrm{X} 3$ & Triệu đồng \\
\hline
\end{tabular}

\subsection{Các yếu tố tác động đến hiệu quả của các DNBĐSVN}

Nguồn: Tổng hợp và đề xuất của tác giả

Dựa trên khung lý thuyết về các yếu tố tác động đến hiệu quả của các doanh nghiệp BĐS, bối cảnh thực tiển và nguồn dữ liệu sẵn có của Việt Nam, tác giả đề xuất 18 yếu tác động đến hiệu quả của các DNBĐSVN (Bảng 2). Trong đó, ngoài 13 yếu tố được sử dụng theo các nghiên cứu trước, tác giả bổ sung thêm 5 biến mới gồm: rủi ro hệ thống, tính dễ tổn thương của khu vực ngân hàng, hiệu quả Chính phủ, giá $\mathrm{BĐS}$ và chỉ số tiếp cận đất đai dựa trên các quan điểm tương ứng của Sharpe (1964), Lintner (1965), You \& Zi, (2007), Kuppuswamy \& cộng sự (2014); Klimczak (2010), Lieser \& Groh (2014). Nguồn dữ liệu thứ cấp được thu thập từ các báo cáo tài chính (BCTC) đã kiểm toán của 38 DNBĐSVN niêm yết trên HOSE, Thống kê tài chính quốc tế của Quỹ Tiền tệ Quốc tế (IFS), Tổng Cục Thống kê Việt Nam (GSO), Worldwide Governance Indicators (WGI), Phòng Thương mại \& Công nghiệp Việt Nam (VCCI) và tính toán của tác giả trong giai đoạn 2010-2019.

Bảng 2: Các yếu tố tác động đến hiệu quả của các DNBĐSVN

\begin{tabular}{|c|c|c|c|c|}
\hline Biến & Ký hiệu & Cách đo lường & Kỳ vọng & $\begin{array}{l}\text { Nguồn dũ̃ } \\
\text { liệu }\end{array}$ \\
\hline \multicolumn{5}{|l|}{ Hiệu quả của DNBĐSVN } \\
\hline $\begin{array}{l}\text { Hiệu quả kỹ thuật với biên sản xuất } \\
\text { cố định theo quy mô }\end{array}$ & CRSTE & Ước lượng từ DEA & & BCTC \\
\hline $\begin{array}{l}\text { Hiệu quả kỹ thuật với biên sản xuất } \\
\text { biến đồi theo quy mô }\end{array}$ & VRSTE & Ước lượng từ DEA & & BCTC \\
\hline Hiệu quả theo quy mô & SE & Ước lượng từ DEA & & BCTC \\
\hline \multicolumn{5}{|l|}{ Yếu tố đặc thù doanh nghiệp } \\
\hline Qui mô doanh nghiệp & SIZE & $\begin{array}{l}\text { Logarithm tự nhiên của tổng } \\
\text { tài sản }\end{array}$ & + & BCTC \\
\hline Khả năng thanh toán & LIQ & $\begin{array}{l}\text { Khả năng thanh toán nhanh = } \\
\text { (Tài sản ngắn hạn-Hàng tồn } \\
\text { kho)/Nợ ngắn hạn }\end{array}$ & + & BCTC \\
\hline Vòng quay tài sản & ATR & Doanh thu thuần/Tổng tài sản & + & $\mathrm{BCTC}$ \\
\hline Khả năng sinh lời & ROA & $\begin{array}{l}\text { Lợi nhuận sau thué/Tổng tài } \\
\text { sản }\end{array}$ & + & BCTC \\
\hline Tốc độ tăng trưởng & GROWTH & $\begin{array}{l}\text { Tăng trưởng doanh thu: Phần } \\
\text { trăm thay đồi doanh thu hàng } \\
\text { năm }\end{array}$ & + & BCTC \\
\hline
\end{tabular}



NIÊM YÊT TẠI VIỆT NAM

\begin{tabular}{|c|c|c|c|c|}
\hline Biến & Ký hiệu & Cách đo lường & Kỳ vọng & $\begin{array}{l}\text { Nguồn dữ } \\
\text { liệu }\end{array}$ \\
\hline Cấu trúc vốn & $\mathrm{CS}$ & Tổng nợ/Tổng tài sản & - & BCTC \\
\hline Tuổi của doanh nghiệp & AGE & $\begin{array}{l}\text { Số năm hoạt động của doanh } \\
\text { nghiệp }\end{array}$ & + & BCTC \\
\hline Sở hữu nhà nước & $\mathrm{SO}$ & $\begin{array}{l}\text { Biến giả, nhận giá trị́ } 1 \text { nếu là } \\
\text { doanh nghiệp có vốn đầu tư } \\
\text { Nhà nước và } 0 \text { nếu ngược lại }\end{array}$ & - & BCTC \\
\hline Sở hữu nước ngoài & $\mathrm{FO}$ & $\begin{array}{l}\text { Biến giả, nhận giá trị́ } 1 \text { nếu là } \\
\text { doanh nghiệp có vốn đầu tư } \\
\text { nước ngoài và } 0 \text { nếu ngược } \\
\text { lại }\end{array}$ & + & BCTC \\
\hline Rủi ro hệ thống & BETA & $\begin{array}{l}\text { Hệ số Beta theo cách tiếp cận } \\
\text { mô hình CAPM của Sharpe } \\
\text { (1964) \& Lintner (1965) }\end{array}$ & - & $\begin{array}{l}\text { Tính toán } \\
\text { của tác giả }\end{array}$ \\
\hline \multicolumn{5}{|l|}{ Yếu tố đặc thù ngành } \\
\hline Giá bất động sản & REP & Chỉ số giá bất động sản & + & GSO \\
\hline Chỉ số tiếp cận đất đai & LA & $\begin{array}{l}\text { Chỉ số tiếp cận đất đai bình } \\
\text { quân của } 63 \text { tỉnh/thành phố }\end{array}$ & + & VCCI \\
\hline \multicolumn{5}{|l|}{ Yếu tố vĩ mô } \\
\hline Tăng trưởng kinh tế & GDP & Tăng trưởng GDP & + & GSO \\
\hline Lãi suất & LDRR & $\begin{array}{l}\text { Chênh leẹch lãi suất cho vay } \\
\text { và lãi suất tiền gửi }\end{array}$ & - & IFS \\
\hline Lạm phát & INF & Tăng trưởng CPI & - & IFS \\
\hline Tỷ giá & NER & $\begin{array}{l}\text { Logarithm tự nhiên của tỷ giá } \\
\text { USD/VND }\end{array}$ & - & IFS \\
\hline $\begin{array}{l}\text { Tính dễ tổn thương của khu vực } \\
\text { ngân hàng }\end{array}$ & $\mathrm{BSF}$ & $\begin{array}{l}\text { Chỉ số dễ tổn thương của khu } \\
\text { vực ngân hàng theo nghiên } \\
\text { cứu của Kibritcioglu }(2003)^{1}\end{array}$ & + & $\begin{array}{l}\text { Tính toán } \\
\text { của tác giả }\end{array}$ \\
\hline Hiệu quả Chính phủ & GE & Chỉ số hiệu quả Chính phủ & + & WGI \\
\hline
\end{tabular}

\subsection{Mô hình nghiên cứu các yếu tố tác động đến hiệu quả của các DNBĐSVN}

Nguồn: Tổng hợp và đề xuất của tác giả

Để xác định các yếu tố ảnh hưởng đến hiệu quả kỹ thuật và hiệu quả theo quy mô của các DNBĐSVN, tác giả sử dụng kết hợp hai mô hình Tobit $\& \mathrm{BMA}$ nhằm phát huy những ưu điểm của từng mô hình để đạt được hiệu quả kiểm định cao nhất.

\section{Mô hình Tobit}

Theo Gujarati (2003), mô hình Tobit được sử dụng trong phân tích hồi quy cho trường hợp biến phụ thuộc bị kiểm duyệt như biến hiệu quả của các DNBĐSVN. Do tính chất bị kiểm duyệt của biến phụ thuộc này, nên khi áp dụng các phương pháp bình phương bé nhất thông thường, mô hình tác động cố định hay mô hình tác động ngẫu nhiên sẽ dẫn đến ước tính sai lệch bởi vì không đáp ứng được điều kiện $\mathrm{E}(\mathrm{u})=0$. Do đó, tương tự như Abdullah \& cộng sự (2019), nghiên cứu này cũng sử dụng Tobit để phân tích các yếu tố tác động đến hiệu quả của các DNBĐSVN.

Mô hình hồi quy Tobit có dạng như sau: $\mathrm{Y}^{*}=\beta_{0}+\sum_{i=1}^{n} \beta_{i} X_{i}+\delta_{i}, \delta_{i} \sim \mathrm{N}\left(0, \sigma^{2}\right)$ với $0<\mathrm{Y}^{*}<1$

${ }^{1} \mathrm{BSF}=\frac{\left(\frac{C P S_{t}-\mu_{C P S}}{\sigma_{C P S}}\right)+\left(\frac{F L_{t}-\mu_{F L}}{\sigma_{F L}}\right)+\left(\frac{D E P_{t}-\mu_{D E P}}{\sigma_{D E P}}\right)}{3}$

Trong đó: CPS, FL, DEP tương ứng là phần trăm thay đổi tín dụng thực của hệ thống ngân hàng đối với khu vực tư nhân theo năm ; FL là phần trăm thay đổi nợ nước ngoài thực của hệ thống ngân hàng theo năm ; DEP là phần trăm thay đổi tổng tiền gửi thực theo năm của hệ thống ngân hàng; $\mu$ và $\sigma$ tương ứng là trung bình số học và độ lệch chuẩn của $\mathrm{CPS}, \mathrm{FL}, \mathrm{DEP}$ tương ứng 


$$
\begin{cases}Y=Y^{*}, & Y^{*}>0 \\ Y=0, & Y^{*}<0\end{cases}
$$

Trong đó:

$\mathrm{Y}_{\mathrm{i}}^{*}$ là các biến phụ thuộc phản ánh hiệu quả của các DNBĐSVN được ước lượng bằng phương pháp hợp lý cực đại.

$\mathrm{Y}_{\mathrm{i}}$ là biến phụ thuộc phản ánh hiệu quả của các DNBĐSVN, được đo lường từ phương pháp DEA, bao gồm CRSTE, VRSTE và SE.

$\mathrm{X}_{\mathrm{i}}$ là các biến độc lập phản ánh 18 yếu tố tác động đến hiệu quả của các DNBĐSVN được mô tả trong Bảng 2.

$\beta_{\mathrm{i}}$ là các tham số được ước lượng; $\delta_{\mathrm{i}}$ là sai số theo phân phối chuẩn.

\section{Mô hình BMA}

Mô hình BMA được phát triển bởi Madigan \& York (1995), sử dụng phương pháp chuỗi so sánh Markov Monte Carlo, có thể được ứng dụng để phát hiện các yếu tố ảnh hưởng mạnh nhất đến hiệu quả của các DNBĐSVN từ tập hợp các yếu tố tiềm năng. Khi sử dụng BMA trong phân tích hồi quy cho trường hợp biến phụ thuộc bị kiểm duyệt như biến hiệu quả của các DNBĐSVN chẳng những sẽ không gặp phải nhược điểm như các phương pháp hồi quy thông thường mà cách tiếp cận này còn có có điểm vượt trội hơn so với Tobit là có thể xếp hạng các các yếu tố tác động đến hiệu quả các DNBĐSVN theo tiên lượng tương đối của từng yếu tố. Do đó, để đảm bảo tính vững của kiểm định, cùng với Tobit, nghiên cứu này cũng sử dụng BMA để phân tích các yếu tố tác động đến hiệu quả của các DNBĐSVN.

Mô hình hồi quy tuyến tính được xem xét như sau: $Y=\alpha_{y}+X_{y} \beta_{y}+\varepsilon \quad \varepsilon \sim\left(0, \sigma^{2} I\right)$

Trong đó:

Y là biến phụ thuộc phản ánh hiệu quả của các DNBĐSVN được đo lường từ phương pháp DEA, bao gồm CRSTE, VRSTE và SE.

$\alpha_{y}$ là một hằng số; $\beta_{y}$ là một véc tơ các hệ số; $\varepsilon$ là sai số nhiễu trắng ;

X $\gamma$ biểu thị một số tập hợp con của tất cả các biến độc lập có sẵn liên quan, tức là 18 yếu tố tiềm năng tác động đến hiệu quả của các DNBĐSVN được mô tả trong Bảng 2.

Với $\mathrm{k}$ biến độc lập tiềm năng cho $2^{\mathrm{k}}$ mô hình tiềm năng. Giá trị trung bình của mô hình BMA được tính toán từ thông tin của các mô hình tiềm năng trên cơ sở sử dụng xác suất hậu nghiệm được thực hiện bởi Định lý Bayes như sau: $\mathrm{P}(\mathrm{M} \gamma \mid \mathrm{y}, \mathrm{X}) \alpha P(y \mid M \gamma, X) * P\left(M_{k} \mid Y\right)$

Trong đó: $\mathrm{P}(\mathrm{M} \gamma \mid \mathrm{y}, \mathrm{X})$ là xác suất hậu nghiệm của mô hình. Sự vững mạnh của một biến giải thích được thể hiện bởi xác suất hậu nghiệm thu nhận (Posterior Inclusion Probability -PIP) và biến với một PIP cao $(>0,5)$ được coi là yếu tố quyết định của biến phụ thuộc.

\section{KẾT QUẢ NGHIÊN CỨU VÀ THẢO LUÂN}

\subsection{Hiệu quả của các DNBĐSVN trong giai đoạn 2010-2019}

Kết quả ước lượng DEA tại Bảng 3 cho thấy trong giai đoạn 2010-2019, hiệu quả theo quy mô trung bình của các DNBĐSVN ở mức $93,01 \%$, với hiệu quả kỹ thuật đạt được từ biên sản xuất cố định theo quy mô là $80,50 \%$ và hiệu quả kỹ thuật đạt được từ biên sản xuất thay đổi theo quy mô là $86,61 \%$. Kết quả này cho thấy trong thời gian qua để tạo ra cùng một mức sản lượng đầu ra như nhau thì các DNBĐSVN đã chưa sử dụng tối đa các nguồn lực đầu vào và còn lãng phí các đầu vào ở mức trung bình từ $15,46 \%$ đến $24,22 \%$. Trong số 38 DNBĐSVN trong mẫu nghiên cứu, 20 doanh nghiệp có hiệu quả theo quy mô cao hơn mức trung bình chung của cả mẫu nghiên cứu, 10 doanh nghiệp có hiệu quả theo quy mô thấp hơn mức trung bình chung của cả mẫu nghiên cứu gồm DRH, DTA, ITA, KDH, LGL, PDR, PPI, SZL, VIC, VPH. Hình 2 cho thấy từ năm 2017, xu hướng của các đường hiệu quả bắt đầu giảm dần, sau đợt gia tăng từ năm 2013 trong bối cảnh nền kinh tế Việt Nam bắt đầu phục hồi sau ảnh hươ̛ng của cuộc khủng hoảng tài chính toàn cầu 2007-2008. Xét trong toàn giai đoạn nghiên cứu, năm 2010, 2013, 2019 là các năm các DNBĐSVN có hiệu quả theo qui mô thấp hơn mức trung bình chung, trong đó năm 2019 là năm các DNBĐSVN có hiệu quả theo qui mô thấp nhất ở mức $83,9 \%$. 

NIÊM YẾT TẠI VIỆT NAM

Bảng 3: Hiệu quả kỹ thuật và hiệu quả theo quy mô của các DNBĐSVN trong giai đoạn 2010-2019

\begin{tabular}{|c|c|c|c|}
\hline Doanh nghiệp & CRSTE & VRSTE & SE \\
\hline CLG & 0,7988 & 0,8697 & 0,9199 \\
\hline D2D & 0,8917 & 0,9351 & 0,9512 \\
\hline DIG & 0,7390 & 0,7671 & 0,9649 \\
\hline DLG & 0,7509 & 0,7698 & 0,9765 \\
\hline DRH & 0,8134 & 0,9308 & 0,8762 \\
\hline DTA & 0,6209 & 1,0000 & 0,6209 \\
\hline $\mathrm{DXG}$ & 0,9603 & 0,9926 & 0,9668 \\
\hline HAG & 0,8405 & 0,9660 & 0,8664 \\
\hline $\mathrm{HDC}$ & 0,8215 & 0,8498 & 0,9663 \\
\hline HDG & 0,8726 & 0,9065 & 0,9647 \\
\hline HQC & 0,7610 & 0,7705 & 0,9858 \\
\hline IJC & 0,8258 & 0,8665 & 0,9502 \\
\hline ITA & 0,8045 & 0,8664 & 0,9294 \\
\hline ITC & 0,6284 & 0,6580 & 0,9517 \\
\hline $\mathrm{KAC}$ & 0,7670 & 0,9767 & 0,7832 \\
\hline $\mathrm{KBC}$ & 0,8936 & 0,9242 & 0,9653 \\
\hline $\mathrm{KDH}$ & 0,7271 & 0,8287 & 0,8885 \\
\hline $\mathrm{LCG}$ & 0,7872 & 0,8071 & 0,9737 \\
\hline LGL & 0,7768 & 0,8408 & 0,9207 \\
\hline LHG & 0,9288 & 0,9503 & 0,9765 \\
\hline $\mathrm{NBB}$ & 0,8582 & 0,8830 & 0,9709 \\
\hline NTL & 0,9590 & 0,9763 & 0,9816 \\
\hline NVT & 0,8021 & 0,8357 & 0,9551 \\
\hline OGC & 0,8079 & 0,8377 & 0,9637 \\
\hline PDR & 0,8133 & 0,8972 & 0,9065 \\
\hline PPI & 0,5926 & 0,7768 & 0,7667 \\
\hline QCG & 0,6778 & 0,6992 & 0,9694 \\
\hline REE & 0,9261 & 0,9831 & 0,9391 \\
\hline SC5 & 0,8908 & 0,9036 & 0,9847 \\
\hline SCR & 0,7658 & 0,7911 & 0,9695 \\
\hline SJS & 0,7661 & 0,7833 & 0,9523 \\
\hline SZL & 0,8762 & 0,9414 & 0,9294 \\
\hline TDC & 0,7502 & 0,7758 & 0,9660 \\
\hline $\mathrm{TDH}$ & 0,7849 & 0,8121 & 0,9698 \\
\hline UDC & 0,7128 & 0,7475 & 0,9524 \\
\hline UIC & 1,0000 & 1,0000 & 1,0000 \\
\hline VIC & 0,8947 & 1,0000 & 0,8947 \\
\hline VPH & 0,7032 & 0,7949 & 0,8793 \\
\hline Trung bình & $\mathbf{0 , 8 0 5 0}$ & 0,8661 & 0,9302 \\
\hline
\end{tabular}




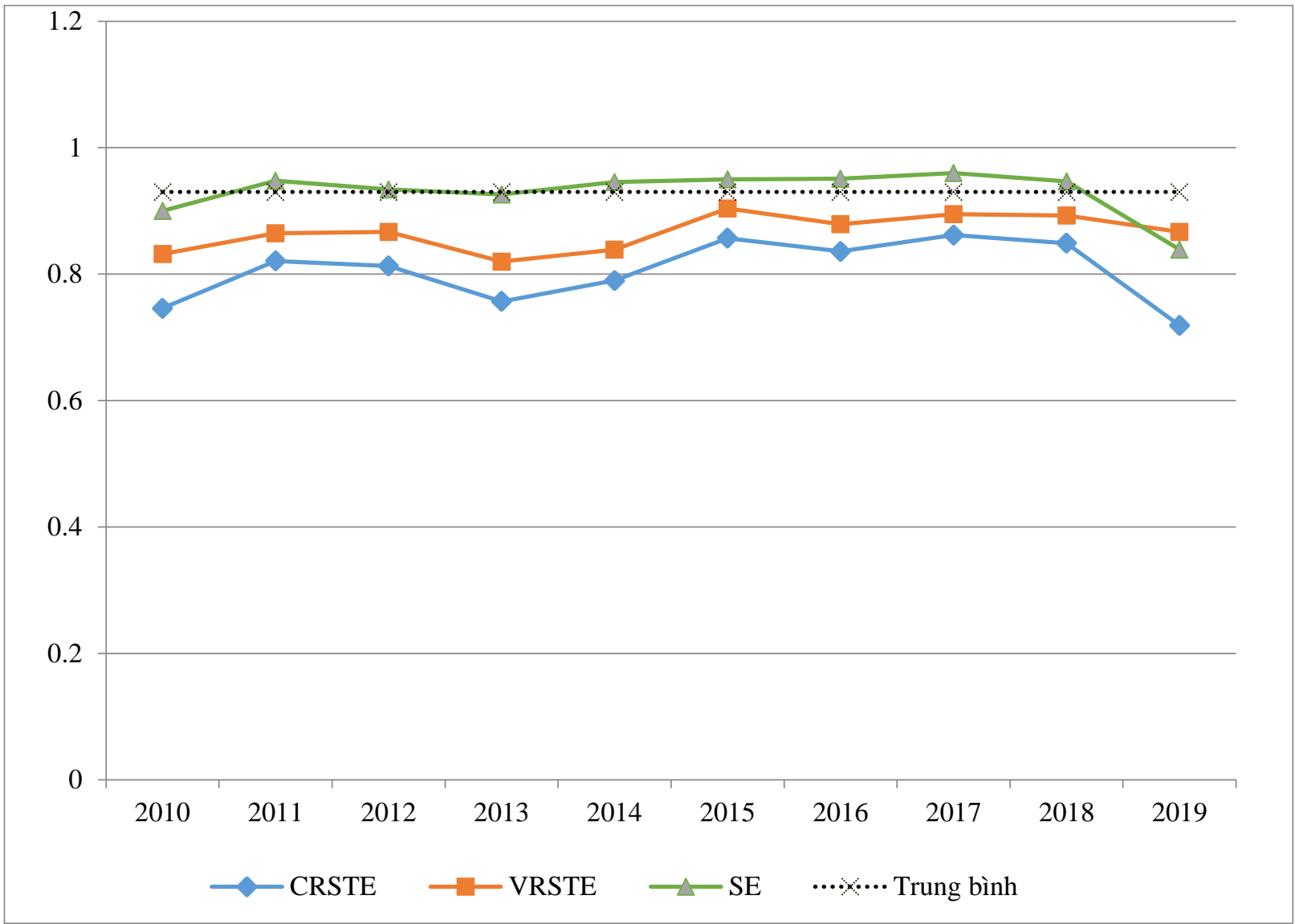

Hình 2: Hiệu quả kỹ thuật và hiệu quả theo quy mô của các DNBĐSVN trong giai đoạn 2010-2019

\subsection{Kiểm định các yếu tố tác động đến hiệu quả của các DNBĐSVN}

Nguồn: Tính toán của tác giả tù̀ DEAP2.1

Kết quả ước lượng ba mô hình Tobit theo các biến phụ thuộc CRSTE, VRSTE và SE tại Bảng 4 cho thấy hiệu quả của các DNBĐSVN chịu ảnh hưởng của 13 yếu tố, gồm: SIZE, ATR, ROA, CS, FO, BETA, REP, LA, GDP, LDRR, INF, BSF, GE tại mức ý nghĩa thống kê $1 \%, 5 \%, 10 \%$ và dấu của các hệ số ước lượng đều phù hợp với kỳ vọng ban đầu.

Bảng 4: Kết quả ước lượng Tobit về các yếu tố tác động đến hiệu quả của các DNBĐSVN trong giai đoạn 2010-2019

\begin{tabular}{|c|c|c|c|}
\hline Biến & CRSTE & VRSTE & SE \\
\hline SIZE & $0,0034^{*}$ & 0,0045 & $0,0035^{*}$ \\
& $(0,0089)$ & $(0,0176)$ & $0,0074)$ \\
\hline LIQ & 0,0106 & 0,0038 & 0,0036 \\
& $(0,0095)$ & $(0,0103)$ & $0,0069)$ \\
\hline ATR & $0,0445^{* *}$ & 0,0275 & $(0,0108)$ \\
\hline ROA & $(0,0134)$ & $(0,0154)$ & $0,6768^{* * *}$ \\
& $1,0333^{* * *}$ & $0,4362^{* * *}$ & $(0,0736)$ \\
\hline GROWTH & $(0,1132)$ & $(0,1098)$ & 0,0014 \\
& 0,0021 & 0,0035 & $(0,0012)$ \\
\hline CS & $(0,0019)$ & $(0,0018)$ & $-0,0031^{* *}$ \\
& $-0,0256^{* *}$ & $-0,0374 *$ & $(0,0392)$ \\
\hline AGE & $(0,0536)$ & $(0,0569)$ & 0,0024 \\
& 0,0002 & 0,0026 & $(0,0013)$ \\
\hline SO & $(0,0014)$ & $(0,0017)$ & $-0,0075$ \\
& $-0,0342$ & $-0,0383$ & $(0,0215)$ \\
\hline FO & $(0,0225)$ & $(0,0291)$ & $0,0597^{* * *}$ \\
& $0,0513^{* * *}$ & $0,0385^{* *}$ & $(0,0225)$ \\
\hline BETA & $(0,0287)$ & $(0,0320)$ & $-0,0202^{* *}$ \\
\hline
\end{tabular}



NIÊM YÊT TẠI VIẸTT NAM

\begin{tabular}{|c|c|c|c|}
\hline Biến & CRSTE & VRSTE & SE \\
\hline & $(0,0180)$ & $(0,0176)$ & $(0,0118)$ \\
\hline REP & $\begin{array}{c}0,0303 * * * \\
(0,0417)\end{array}$ & $\begin{array}{c}0,0306 * * \\
(0,0388)\end{array}$ & $\begin{array}{c}0,0056 * * \\
(0,0259)\end{array}$ \\
\hline LA & $\begin{array}{c}0,0287 * * * \\
(0,0417)\end{array}$ & $\begin{array}{c}0,0143 * * * \\
(0,0378)\end{array}$ & $\begin{array}{c}0,0167 * * * \\
(0,0259)\end{array}$ \\
\hline GDP & $\begin{array}{c}0,00160 * * \\
(0,0574)\end{array}$ & $\begin{array}{c}0,0041 * * * \\
(0,0534)\end{array}$ & $\begin{array}{c}0,0201 * * * \\
(0,0357)\end{array}$ \\
\hline LDRR & $\begin{array}{c}-0,0383 * * * \\
(0,0328)\end{array}$ & $\begin{array}{c}-0,0025 * * * \\
(0,0305)\end{array}$ & $\begin{array}{c}-0,0424 * * * \\
(0,0204)\end{array}$ \\
\hline INF & $\begin{array}{c}-0,0272 * * * \\
(0,0189)\end{array}$ & $\begin{array}{c}-0,0022 * * \\
(0,0176)\end{array}$ & $\begin{array}{c}-0,0275^{* * * *} \\
(0,0118)\end{array}$ \\
\hline NER & $\begin{array}{l}-0,0443 \\
(0,0268)\end{array}$ & $\begin{array}{l}-0,0162 \\
(0,0249)\end{array}$ & $\begin{array}{l}-0,0285 \\
(0,0166)\end{array}$ \\
\hline BSF & $\begin{array}{l}0,2439 * \\
(0,1432)\end{array}$ & $\begin{array}{c}0,1165^{* *} \\
(0,1335)\end{array}$ & $\begin{array}{c}0,1270 * * \\
(0,0893)\end{array}$ \\
\hline GE & $\begin{array}{c}0,4616 * * * \\
(0,2221)\end{array}$ & $\begin{array}{c}0,29991 * * * \\
(0,2060)\end{array}$ & $\begin{array}{c}0,2130 * * * \\
(0,1376)\end{array}$ \\
\hline $\mathrm{C}$ & $\begin{array}{c}-2,1806 * * * \\
(4,2421)\end{array}$ & $\begin{array}{c}-2,1685 * * * \\
(3,9439)\end{array}$ & $\begin{array}{c}-1,6323 * * * \\
(2,6332)\end{array}$ \\
\hline
\end{tabular}

Ghi chú: *, **, *** Ý nghĩa thống kê lần lượt ở múc 10\%, 5\% và 1\% Nguồn: Tính toán của tác giả tù Stata 14

Kết quả ước lượng BMA tại Bảng 5 được tổng hợp từ cách sử dụng 10.000 lần lặp lại của chuỗi Markov Monte Carlo cho thấy 11 yếu tố tác động mạnh đến hiệu quả của các DNBĐSVN với $\mathrm{PIP}>0,5$, bao gồm ROA, CS, FO, BETA, REP, LA, GDP, LDRR, INF, BSF, GE. Đặc biệt, ROA, FO, LA, GE, LDRR, là 5 yếu tố tác động mạnh mẽ nhất với PIP dao động từ $0,9-1,0$ ở tất cả ba mô hình theo các biến phụ thuộc CRSTE, VRSTE và SE. Ngoài ra, kết quả BMA cũng chỉ ra xác suất tích lũy của ba mô hình lần lượt ở các mức 0,$76 ; 0,82 ; 0,87$ cho thấy mô hình đạt mức độ tin cậy cao.

Bảng 5: Kết quả ước lượng BMA về các yếu tố tác động đến hiệu quả của các DNBĐSVN trong giai đoạn 2010-2019

\begin{tabular}{|c|c|c|c|c|c|c|}
\hline \multirow{2}{*}{ Biến/Chỉ tiêu } & \multicolumn{2}{|c|}{ CRSTE } & \multicolumn{2}{c|}{ VRSTE } & \multicolumn{2}{c|}{ SE } \\
\cline { 2 - 7 } & PIP & Post Mean & PIP & Post Mean & PIP & Post mean \\
\hline SIZE & 0,4164 & 0,0002 & 0,4557 & 0,0006 & 0,4207 & 0,0009 \\
\hline LIQ & 0,2055 & 0,0007 & 0,2258 & 0,0035 & 0,2352 & 0,0008 \\
\hline ATR & 0,4876 & 0,0048 & 0,4175 & 0,0407 & 0,4316 & 0,0035 \\
\hline ROA & 1,0000 & 0,6098 & 1,0000 & 1,1065 & 1,0000 & 0,5230 \\
\hline GROWTH & 0,3458 & 0,0002 & 0,3000 & 0,0003 & 0,3361 & 0,0001 \\
\hline CS & 0,5344 & $-0,0021$ & 0,5357 & $-0,0256$ & 0,5409 & 0,0004 \\
\hline AGE & 0,2892 & 0,7224 & 0,1576 & 0,0004 & 0,4590 & 0,0008 \\
\hline SO & 0,3980 & $-0,0078$ & 0,4062 & $-0,0180$ & 0,3358 & 0,0002 \\
\hline FO & 0,9196 & 0,0003 & 0,9584 & 0,0685 & 1,0000 & 0,1057 \\
\hline BETA & 0,7452 & $-0,0652$ & 0,7563 & $-0,0018$ & 0,6933 & 0,0046 \\
\hline REP & 0,6269 & 0,0002 & 0,6579 & 0,0231 & 0,6786 & 0,0011 \\
\hline LA & 0,9117 & 0,0074 & 0,9998 & 0,0660 & 0,9449 & 0,0108 \\
\hline GDP & 0,7248 & 0,0004 & 0,7294 & 0,0456 & 0,7619 & 0,0232 \\
\hline LDRR & 0,9146 & $-0,0014$ & 0,9935 & $-0,0408$ & 0,9214 & 0,0185 \\
\hline INF & 0,7177 & $-0,0013$ & 0,7008 & $-0,0038$ & 0,7437 & 0,0004 \\
\hline NER & 0,2184 & $-0,0001$ & 0,2935 & $-0,0737$ & 0,3204 & $-0,0505$ \\
\hline BSF & 0,6326 & 0,0011 & 0,5866 & 0,0784 & 0,6656 & 0,0005 \\
\hline GE & 0,9419 & 0,0049 & 1,0000 & 0,6515 & 1,0000 & 0,2210 \\
\hline Xác suất tích lũy & & $\mathbf{0 , 7 6}$ & & $\mathbf{0 , 8 7}$ & $\mathbf{0 , 8 2}$ \\
\hline
\end{tabular}

Nguồn: Tính toán của tác giả tù phần mềm $R$

Như vậy, tổng hợp kết quả ước lượng từ các mô hình Tobit và BMA đã chỉ ra 13 yếu tố tác động đến hiệu quả của các DNBĐSVN, bao gồm: Qui mô doanh nghiệp, vòng quay tài sản, khả năng sinh lời, sở hữu nước ngoài, giá $\mathrm{BĐS}$, vấn đề tiếp cận đất đai, tăng trưởng kinh tế, tính dễ tổn thương của khu vực ngân hàng và 
hiệu quả Chính phủ có tác động cùng chiều, trong khi đó rủi ro hệ thống, cấu trúc vốn, lãi suất và lạm phát có tác động ngược chiều đến hiệu quả của các DNBĐSVN. Trong đó, năm biến mới gồm: Rủi ro hệ thống, tính dễ tổn thương của khu vực ngân hàng, hiệu quả Chính phủ, giá BĐS và vấn đề tiếp cận đất đai đều có tác động mạnh mẽ đến hiệu quả của các DNBĐSVN với mức ý nghĩa thống kê và PIP cao.

Kết quả này phù hợp với kỳ vọng của tác giả và tương đồng với kết quả nghiên cứu của Huệ \& Lâm (2017), You \& Zi (2007), Abdullah \& cộng sự (2019), Wei \& cộng sự (2004), Saul \& cộng sự (2008) và phù hợp với quan điểm của Sharpe (1964), Lintner (1965), Kuppuswamy \& cộng sự (2014), Klimczak (2010), Lieser \& Groh (2014).

\section{KẾT LUẬN VÀ KHUYẾN NGH!}

\subsection{Kết luận}

Bài viết này nghiên cứu các yếu tố tác động đến hiệu quả kỹ thuật và hiệu quả theo quy mô của 38 DNBĐSVN trong giai đoạn 2010-2019. Đầu tiên, nghiên cứu đo lường hiệu quả của các DNBĐSVN trong giai đoạn 2010-2019 theo cách tiếp cận DEA. Sau đó, nghiên cứu sử dụng kết hợp hai mô hình Tobit và BMA để xem xét các yếu tố tác động đến hiệu quả của các DNBĐSVN. Kết quả ước lượng DEA cho thấy: Trong giai đoạn 2010-2019, hiệu quả theo quy mô trung bình của các DNBĐSVN ở mức 93,01\%, với hiệu quả kỹ thuật đạt được từ biên sản xuất cố định theo quy mô là $80,50 \%$ và hiệu quả kỹ thuật đạt được từ biên sản xuất thay đổi theo quy mô là $86,61 \%$, phản ánh rằng các DNBĐSVN chưa sử dụng tối đa các nguồn lực đầu vào. Kết quả mô mình Tobit và $B M A$ chỉ ra rằng hiệu quả của các DNBĐSVN chịu ảnh hưởng của 13 yếu tố, bao gồm: Qui mô doanh nghiệp, vòng quay tài sản, khả năng sinh lời, sở hữu nước ngoài, giá BĐS, chỉ số tiếp cận đất đai, tăng trưởng kinh tế, tính dễ tổn thương của khu vực ngân hàng và hiệu quả Chính phủ có tác động cùng chiều, trong khi đó rủi ro hệ thống, cấu trúc vốn, lãi suất và lạm phát có tác động ngược chiều đến hiệu quả của các DNBĐSVN. Nghiên cứu này đã mang lại đóng góp mới về phương pháp tiếp cận tại Việt Nam trên cơ sở lần đâu tiên sử dụng cách tiếp cận DEA để đo lường hiệu quả của các DNBĐSVN và sử dụng kết hợp hai mô hình Tobit và BMA để xem xét các yếu tố tác động đến hiệu quả của các DNBĐSVN. Kết quả nghiên cứu đã bổ sung những bằng chứng thực nghiệm vào khung lý thuyết về các yếu tố tác động đến hiệu quả doanh nghiệp nói chung và hiệu quả của các doanh nghiệp $\mathrm{BĐS}$ nói riêng.

\subsection{Khuyến nghị}

Dựa trên kết quả nghiên cứu về các yếu tố tác động đến hiệu quả của các DNBĐSVN, tác giả đề xuất một số khuyến nghị nhằm nâng cao hiệu quả tại các DNBĐSVN trong thời gian tới như sau:

5.2.1 Khuyến nghị đối với các DNBĐSVN

- Thực hiện tối ưu hóa quy trình kinh doanh, tăng trưởng quy mô nhân sự, tăng cường đầu tư đổi mới công nghệ nhằm mở rộng quy mô hoạt động kinh doanh.

- Thực hiện tái cẩu trúc vốn hiệu quả nhằm đảm bảo tối thiểu hóa chi phí sử dụng vốn bình quân, tối đa hóa lợi nhuận và nâng cao hiệu quả doanh nghiệp. Theo đó, các DNBĐSVN cần lựa chọn cơ cấu vốn tối ưu theo hướng tăng vốn chủ sở hữu, giảm nợ vay để hạn chế tác động của đòn bẩy tài chính, điều chỉnh giảm việc thanh toán cổ tức bằng tiền mặt, thay vào đó chọn giải pháp thanh toán cổ tức bằng cổ phiếu nhằm gia tăng lợi nhuận giữ lại, tăng cường huy động vốn thông qua phát hành cổ phiếu; tận dụng các khoản ứng trước của khách hàng từ việc bán, cho thuê của các dự án BĐS.

- Tăng cường công tác quản trị rủi ro hệ thống theo hướng xây dựng lại quy trình quản trị rủi ro dựa nền tảng các chuẩn mực quốc tế. Theo đó, tất cả các bộ phận trong doanh nghiệp đều phải tham gia vào quá trình này, theo dõi chặt chẽ biến động rủi ro trong quá khứ, dự báo xu hướng tương lai để có thể kịp thời nhận diện, hạn chế và phòng ngừa rủi ro có khả năng xảy ra tại doanh nghiệp.

- Nâng cao tỷ lệ sở hữu nước ngoài tại các DNBĐSVN trên cơ sở xây dựng chiến lược kinh doanh hiệu quả nhằm tăng cường thu hút đầu tư nước ngoài.

5.2.2 Khuyến nghị đối với các nhà hoạch định chính sách và cơ quan chức năng

- Tiếp tục ổn định kinh tế vĩ mô theo hướng kiểm soát lạm phát, thúc đẩy tăng trưởng kinh tế, ổn định hệ thống ngân hàng, thực hiện chính sách lãi suất linh hoạt, tạo môi trường kinh doanh thuận lợi cho các DNBĐSVN.

- Nâng cao hiệu quả Chính phủ thông qua việc tiếp tục hoàn thiện các văn bản pháp lý liên quan đến hoạt động kinh doanh BĐS như Luật Đất đai, Luật Xây dựng, Luật Nhà ở, Luật Kinh doanh BĐS. 
- Tiếp tục thực hiện việc rà soát, bổ sung quy hoạch đô thị và khu công nghiệp, lập kế hoạch sử dụng đất hợp lý trên cơ sở bảo đảm dành đủ quỹ đất cho phát triển nhà ở xã hội.

- Nâng cao hiệu quả vấn đề tiếp cận đất đai thông qua việc đẩy mạnh cải cách hành chính trong các thủ tục cấp quyền sử dụng đất, rút ngắn thời gian cấp giấy chứng nhận quyền sử dụng đất, quyền sở hữu nhà ở và tài sản khác gắn liền với đất, xây dựng rõ tiêu chí quy trình thu hồi đất theo quy định hiện hành.

- Theo dõi sát tình hình diễn biến thị trường $\mathrm{BĐS}$ để kịp thời có biện pháp bình ổn thị trường, hạn chế tình trạng đầu cơ đất đai, sốt giá và "bong bóng" BĐS.

\section{TÀI LIỆU THAM KHẢO}

[1] Abdullah, M. R. B., Maamor, S., \& Karim, M. Z. A. (2019). Efficiency of real estate firms in Malaysia and its correlates. Int. J Sup. Chain. Mgt Vol, 8(1), 971.

[2] Banker, R. D., Charnes, A., \& Cooper, W. W. (1984). Some models for estimating technical and scale inefficiencies in data envelopment analysis. Management science, 30(9), 1078-1092.

[3] Charnes, A., Cooper, W. W., \& Rhodes, E. (1978). Measuring the efficiency of decision making units. European journal of operational research, 2(6), 429-444.

[4] Coelli, T. (2005). An introduction to Efficiency and Productivity, Spinger Science Business Media, Inc.

[5] Cummins, J. D., Weiss, M. A., Santomero, A. M., David, J., Mary, C., \& Weiss, A. (1999). Analyzing firm performance in the insurance industry using frontier efficiency methods. In Handbook of Insurance Economics.

[6] Cường, P.Đ. \& Tiến, T.Q. (2017). Ảnh hưởng của khác biệt loại hình sở hữu lên hiệu quả hoạt động của các doanh nghiệp Việt Nam. Tạp chí Kinh tế \& Phát triển 2017, số 238, 49-57.

[7] Cvilikas, A., \& Jurkonyte-Dumbliauskiene, E. (2016). Assessment of Risk Management Economic Efficiency Applying Economic Logistic Theory. Transformations in Business \& Economics, 15(3/39), 207-219.

[8] Drucker, Peter F. (1963). Managing for business effectiveness. Harvard Business Review 41: 53-60.

[9] Edgeworth, F. Y. (1881). Mathematical psychics: An essay on the application of mathematics to the moral sciences (Vol. 10). Kegan Paul.

[10] GSO (2019). Niên giám thống Việt Nam năm 2019.

[11] Gujarati, D. (2003). Basic econometrics. McGraw Hill, Boston, USA.

[12] Harris, R. J. (1985). A primer of multivariate statistics. Orlando, FL: Academic Press.

[13] Huệ, N.T.M. \& Lâm, Đ.T. (2017). Tác động của cấu trúc sở hữu đến hiệu quả hoạt động của các công ty niêm yết trên thị trường chứng khoán Việt Nam. Tạp chí Khoa học ĐHQGHN: Kinh tế và Kinh doanh, Tập 33, Số 1 (2017) 23-33.

[14] Hưng, Đ.N \& Mai, V.T.T (2018). Ảnh hưởng của quản trị vốn lưu động đến hiệu quả hoạt động của các công ty niêm yết trên thị trường chứng khoán Việt Nam. Nghiên cứu Kinh tế 2018, số 2, 49-57.

[15] Hương, V.T.T. (2020). Ảnh hưởng của các công ty zombie đến hiệu quả hoạt động tài chính: Kết quả nghiên cứu thực nghiệm trên các công ty niêm yết nhóm ngành vật liệu xây dựng tại Việt Nam. Tạp chí Khoa học Thương mại 2020, số 137-138, 100-108.

[16] Khải, N.Q. \& Toàn, P.N. (2015). Ảnh hưởng của cơ cấu vốn và sở hữu tới hiệu quả hoạt động của các công ty niêm yết. Kinh tế và Dự báo 2015, số Chuyên đề 6, 6-8.

[17] Kibritcioglu, A. (2003). Monitoring Banking Sector Fragility, The Arab Bank Review, vol. 5, no. 2, 51-66.

[18] Klimczak, K. (2010). Determinants of real estate investment. Economics and Sociology, 3(2), 58-66.

[19] Kumar, S., \& Arora, N. (2011). Assessing technical efficiency of sugar industry in Uttar Pradesh: an application of data envelopment analysis. Indian Economic Review, 323-353.

[20] Kuppuswamy, V., Serafeim, G. \& Villalonga, B. (2014). The Effect of Institutional Factors on the Value of Corporate Diversification", Finance and Strategy (Advances in Strategic Management, Vol. 31), Emerald Group Publishing Limited, 37-68.

[21] Lieser, K., \& Groh, A. P. (2014). The determinants of international commercial real estate investment. The Journal of Real Estate Finance and Economics, 48(4), 611-659. 


\section{CÁC YẾU TỐ TÁC ĐộNG ĐẾN HIỆU QUẢ CỦA CÁC DOANH NGHIỆP BÂT ĐộNG SẢN NIÊM YÊTT TẠI VIÊT NAM}

[22] Lintner, J. (1965). Security prices, risk and maximal gains from diversification, Journal of Finance, vol. 20, no. 4, 587-615.

[23] Madigan, D. and York, J. (1995). Bayesian graphical models for discrete data. International Statistical Review $63,215-232$.

[24] MPI (2019). Cục Quản lý đăng ký kinh doanh - Tình hình đăng ký doanh nghiệp năm 2019.

[25] Ngọc, T.T.B \& Trang, P.H (2016). Ảnh hưởng của cấu trúc vốn đến hiệu quả hoạt động của các doanh nghiệp ngành Công nghiệp chế biến, chế tạo niêm yết trên Sở Giao dịch Chứng khoán Thành phố Hồ Chí Minh. Tạp chí Khoa học và Công nghệ. Đại học Đà Nẵng 2016, số 2, 43-47.

[26] Pareto, V. (1927). Manuel d'Economie Politique. Giard et Brier, 2nd Edition.

[27] Phúc, Đ.N. (2018). Ảnh hưởng của cấu trúc vốn đến hiệu quả hoạt động kinh doanh của doanh nghiệp sau cổ phần hóa ở Việt Nam. Tạp chí Nghiên cứu kinh tế 2018, số 1, 11-16.

[28] Ran, M. S., \& Xu, B. (2013). Using a DEA-PNN approach to model the efficiency of real estate public company. Journal of Chongqing University (Social Science Edition), 19(3), 59-64 (in Chinese).

[29] Ross, S. A, Westerfield, R. W., and Jaffe, J. F. (2012). Corporate Finance: Tenth Edition, McGraw-Hill Higher Education.

[30] Saul E., Hanousek, J., Svejnar, J. (2008). The effects of privatization and ownership in transition economies", Journal of Economics Literature, 47 (3), 699-728.

[31] Sharpe, W. F (1964). Capital asset prices: A theory of market equilibrium under conditions of risk, Journal of Finance, vol. 19, no. 3, 425-442.

[32] Shephard, R. W. (1953). Cost and Production Functions. Princeton University Press.

[33] Tabachnick, B. G., Fidell, L. S., \& Ullman, J. B. (2007). Using multivariate statistics (Vol. 5, 481-498). Boston, MA: Pearson.

[34] Thủy, N.T.T (2020). Ảnh hưởng của cấu trúc vốn đến hiệu quả hoạt động kinh doanh của các công ty ngành thủy sản. Tạp chí Tài chính, số 725, 140-143.

[35] Thủy, P.B.G., Tài, T.Đ. \& Anh, T.T.T. (2017). Ảnh hưởng của đặc điểm tổng giám đốc điều hành đến hiệu quả hoạt động doanh nghiệp. Tạp chí Khoa học. Đại học Mở 2017, số 55, 51-63.

[36] Trí, P.M. (2018). Nhân tố ảnh hưởng đến hiệu quả kinh doanh của doanh nghiệp bất động sản niêm yết trên HSX. Tạp chí Tài chính Kỳ 2 - Tháng 02/2018 (675), 93-96.

[37] Trúc, T.T.T \& Thiên, N.Đ. (2015). Ảnh hưởng của chính sách vốn lưu động đến hiệu quả hoạt động của các công ty niêm yết trên thị trường chứng khoán Việt Nam. Khoa học. Đại học Mở 2015, số 3, 101-110.

[38] VARS (2020). Báo cáo Tình hình thị trường bất động sản Việt Nam năm 2020. https://img.vietnamfinance.vn/upload/news/hoanghung_btv/2021/1/12/bao-cao-thi-truong-bds-nam-2020.pdf.

[39] Việt, P.Q \& Mẫn, T.Đ. (2016).Ảnh hưởng của sở hữu gia đình đến hiệu quả hoạt động của các công ty niêm yết. Tạp chí Tài chính. Kỳ 1 2016, số 10, 88-90.

[40] Wang, Y., Zhu, Y., \& Jiang, M. (2015). Efficiency evaluation of listed real estate companies in China. In The Strategies of China's Firms (pp. 89-107). Chandos Publishing.

[41] Wei Z., F. Xie, S. Zhang (2004). Ownership structure and Firm Value in China's Privatized Firms: 1991-2001", Journal of Financial and Quantitative Analysis, 40 (1), 87-108.

[42] Xiao, X. Y. (2014). The evaluation of China's real estate company's operating efficiency by DEA. In Applied Mechanics and Materials (Vol. 644, 5565-5569). Trans Tech Publications Ltd.

[43] You, T., \& Zi, H. (2007). The economic crisis and efficiency change: evidence from the Korean construction industry. Applied Economics, 39(14), 1833-1842.

Ngày nhận bài: 08/12/2020

Ngày chấp nhận đăng: 29/03/2021 